\title{
Frontières
}

\section{L’accompagnement ou la leçon de transcendance avec Yourcenar}

\section{Nathalie Parent}

Volume 17, numéro 1, automne 2004

Au péril de l'accompagnement

URI : https://id.erudit.org/iderudit/1073604ar

DOI : https://doi.org/10.7202/1073604ar

Aller au sommaire du numéro

Éditeur(s)

Université du Québec à Montréal

ISSN

1180-3479 (imprimé)

1916-0976 (numérique)

Découvrir la revue

Citer cet article

Parent, N. (2004). L'accompagnement ou la leçon de transcendance avec Yourcenar. Frontières, 17(1), 35-41. https://doi.org/10.7202/1073604ar
Résumé de l'article

Cet article présente une étude sur le thème de la mort et de la transcendance dans deux nouvelles de Marguerite Yournecar tirées des Nouvelles orientales : La tristesse de Cornélius Berg et Comment Wang-Fô fut sauvé. On cherche ici à mettre en parallèle deux représentations de la mort, des derniers instants de la vie et de l'accompagnement. Une première représentation, celle de La tristesse de Cornélius Berg, met en scène une désacralisation du réel et la solitude dans la mort. Une seconde représentation, celle de Comment Wang-Fô fut sauvé, témoigne de l'apprentissage d'une nouvelle perception du monde et une mort transcendée dans un passage harmonieux et accompagné où le réel se fond à l'art. 
Résumé

Cet article présente une étude sur le thème de la mort et de la transcendance dans deux nouvelles de Marguerite Yournecar tirées des Nouvelles orientales: La tristesse de Cornélius Berg et Comment Wang-Fô fut sauvé. On cherche ici à mettre en parallèle deux représentations de la mort, des derniers instants de la vie et de l'accompagnement. Une première représentation, celle de La tristesse de Cornélius Berg, met en scène une désacralisation du réel et la solitude dans la mort. Une seconde représentation, celle de Comment Wang-Fô fut sauvé, témoigne de l'apprentissage d'une nouvelle perception du monde et une mort transcendée dans un passage harmonieux et accompagné où le réel se fond à l'art.

Mots clés: Marguerite Yourcenar Wanf-Fô - Cornélius Berg accompagnement - transcendance mort.

\section{Abstract}

This article present a thematic study of transcendence in two short stories from Nouvelles orientales by Marguerite Yourcenar: La tristesse de Cornélius Berg and Comment Wang-Fô fut sauvé. Here we compared two representations of death. The first representation which of La tristesse de Cornélius Berg shows the removal of the sacred aura of reality and the solitude in the last moments of life. The second representation which of Comment Wang-Fô fut sauvé shows the learning of a new perception and understanding of the world one of transcendence and harmonious companionship through death.

Key words: Marguerite YourcenarWanf-Fô - Cornélius Berg -

caregiving - transcendence - death

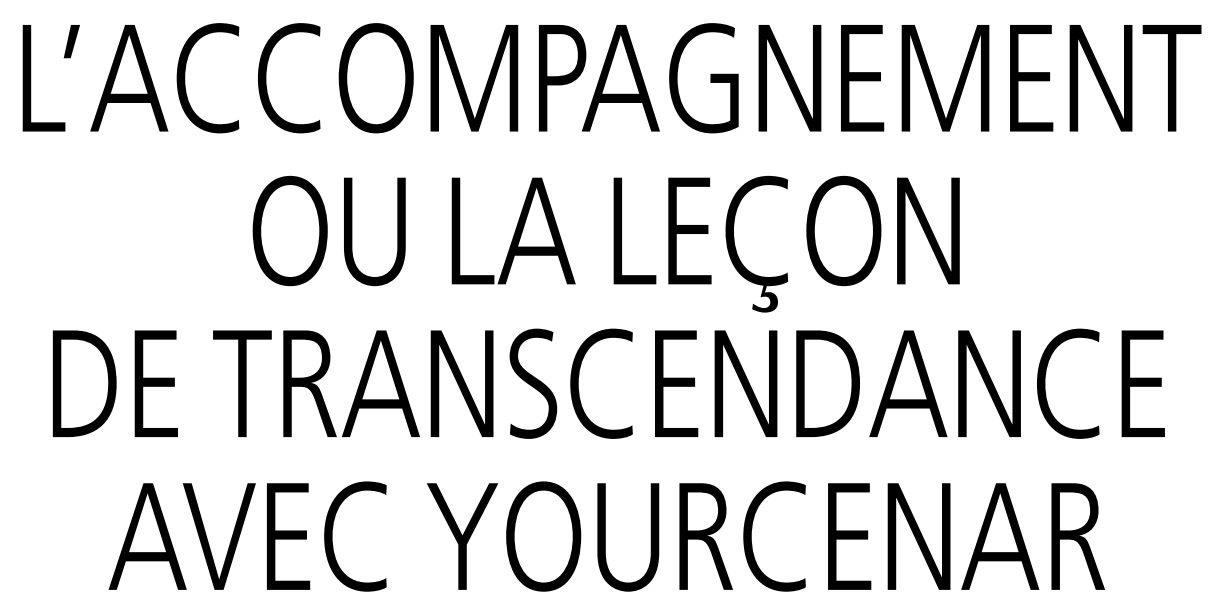

\section{PETITE ÂME, ÂME TENDRE ET FLOTTANTE, COMPAGNE DE MON CORPS, QUI FUT TON HÔTE, TU VAS DESCENDRE DANS CES LIEUX PẤLES, DURS ET NUS, OÙ TU DEVRAS RENONCER AUX JEUX D'AUTREFOIS. UN INSTANT ENCORE, REGARDONS ENSEMBLE LES RIVES FAMILIĖRES, LES OBJETS QUE SANS DOUTE NOUS NE REVERRONS PLUS... TÂCHONS D'ENTRER DANS LA MORT LES YEUX OUVERTS...}

MARGUERITEYOURCENAR, MÉMOIRES D'HADRIEN

\section{Nathalie Parent, \\ chargée de projet, Centre de communication écrite, Université de Montréal.}

Les deux nouvelles de Marguerite Yourcenar: Comment Wang-Fô fut sauvé et La tristesse de Cornélius Berg nous présentent deux conceptions du monde, de la vie et de la mort. Deux univers, deux philosophies; l'une inspirée de l'Orient, l'autre de l'Occident. Deux manières d'appréhender le réel, de le transposer et de le peindre; l'une intégrant le sacré dans la limpidité et l'autre errant dans un dédale de la désacralisation et du questionnement existentiel. Quête de sens, affrontement du non-sens, une vision à ras de terre près de l'humain et de son désordre émotif: voilà Cornélius. Un monde fusionné à la représentation picturale du réel et intégré à la philosophie taoïste, une mort qui vit: voilà Wang-Fô.

Peut-on par la littérature se questionner sur la mort et la sacralisation de la mort? Marguerite Yourcenar l'a fait à maintes reprises. Le sacré et la mort, tout comme l'art, sont des thèmes charnières de l'œuvre. Yourcenar, par le médium qu'est l'histoire, nous invite à réfléchir sur le pouvoir de la contemplation, dernière des sept étapes du rituel de dormition ${ }^{1}$ (De Hennezel et Leloup, 2000, p. 94), et sur le détachement ou la capacité de vivre avec les blessures narcissiques. La conscience de la proximité d'une autre réalité, d'un sens autre de tout objet du réel, est le premier pas vers une conception nouvelle de la mort et vers la capacité de regarder la mort en face, «les yeux ouverts", pour mieux lever le voile:

Le mort n'est pas la fin de la vie, c'est la fin d'une illusion, une délivrance, la délivrance de la souffrance, de l'enchaînement des causes et des effets. C'est pourquoi la mort est un moment béni, le moment le plus sacré de l'existence, car c'est enfin l'occasion d'entrer dans un espace illimité. C'est le moment où la Réalité est enfin révélée (De Hennezel et Leloup, 2000, p. 42).

Nous avons choisi d'étudier ces deux nouvelles pour le contraste qu'elles présentent dans leur conception de la mort et pour les approches de fin de vie différentes qu'elles mettent en scène: une fin accompagnée et l'autre non.

\section{LA TRISTESSE DE CORNÉLIUS BERG}

Cette nouvelle met en scène Cornélius Berg, un peintre de portraits qui vit à Amsterdam au XVII ${ }^{\mathrm{e}}$ siècle, le peintre erre d'auberge en auberge. Cornélius sent sa mort venir: sa capacité à peindre diminue, sa vue diminue, sa main tremble, sa mémoire devient imprécise. Sa fin de vie se déroule dans le mutisme et dans une 
solitude grandissante. Cornélius autrefois le grand voyageur et le bon vivant est maintenant connu comme Cornélius le taciturne.

Alors que Wang-Fô est le peintre de la sérénité, Cornélius Berg est le peintre qui s'enfonce dans l'abîme de la tristesse. Cornélius, à un âge avancé, vit ses derniers moments et c'est en partie par la réflexion qu'il porte sur son passé qu'on découvre le personnage. La tristesse de Cornélius est intimement liée à l'incapacité de peindre qui gagne le peintre et à sa solitude. La tristesse est caractéristique du dépressif. Comme le souligne Julia Kristeva dans Soleil noir (1987), la tristesse est l'humeur fondamentale de la dépression, le chagrin est la manifestation majeure qui trahit le désespéré. La tristesse serait le signal d'un moi primitif blessé, incomplet, vide. Chez le mélancolique, l'identification primaire s'avère être fragile, la tristesse serait l'expression la plus archaïque d'une blessure narcissique, la blessure de l'abandon premier. Le dépressif est aux prises avec les mécanismes de l'identification, il est dans la dialectique complexe de l'idéalisation et de la dévalorisation de soi et de l'autre. Ce qui s'écroule chez le dépressif c'est, entre autres, l'adhésion imaginaire, adhésion qui lui permettrait normalement de faire le deuil de l'objet abandonnique: «Lidentification primaire amorce la compensation de la Chose, en même temps que l'arrimage du sujet à une autre dimension, celle de l'adhésion imaginaire, qui n'est pas sans rappeler le lien de la foi, lequel précisément s'écroule chez le dépressif» (Kristeva, 1987, p. 23).

Aux frontières de la vie et de la mort, le dépressif devient un hyperlucide qui a le sentiment d'être témoin du non-sens de l'Être et de l'absurdité des liens et des êtres. Ce sentiment est coextensif à une perte de la référence, le dépressif se voit dans l'incapacité de produire des enchaînements signifiants autres que rivés au Rien, à la mort:

Le déprimé ne parle de rien, il n'a rien dont parler [...] Cette chose totale et insignifiable est insignifiante: c'est un rien, son Rien, la Mort. Le gouffre qui s'installe entre le sujet et les objets signifiants se traduit par une impossibilité d'enchaînements signifiants. Mais un tel exil révèle un gouffre dans le sujet lui-même (Kristeva, 1987, p. 63).

C'est bien ce qui semble se produire chez Cornélius Berg. La tristesse du peintre est le signe d'une dépression, d'une lente perte de sens qui se lit dans le regard que le peintre porte sur le monde. Le travail visuel de l'artiste est inévitablement touché par la perte de sens. Tout concorde pour que Cornélius se retrouve sur une pente descendante aussi bien psychiquement que physiquement. Cornélius regarde son corps se détériorer: «Par malheur sa main tremblait; il devait ajuster à ses lunettes des verres de plus en plus forts» (p. 1215). Une atmosphère glauque est mise en place dans le texte. L'artiste erre dans des lieux réels ou imaginaires où la luminosité est altérée. Un voile semble tamiser et ternir la clarté qui entoure Cornelius, que ce soit un voile de fumée: "au fond des tavernes enfumées » (p. 1215), ou une forme de poudre: «les pays poudreux de soleil» (p. 1215). La mélancolie affecte le discours de Cornélius: «il tenait alors un discours incompréhensible» (p. 1215), "Ils échangeaient peu de paroles: Cornélius Berg donnait son avis d'un hochement de tête»(p. 1217). Les seules paroles que Cornélius Berg prononce viennent renverser la perception du monde établi: «Dieu est le peintre de l'univers [...]. Quel malheur, monsieur le syndic, que Dieu ne se soit pas borné à la peinture de paysage» (p. 1218). Dans cet énoncé, on note une désacralisation de Dieu qui se traduit par sa personnification. Dieu est réduit à des sentiments humains; Cornélius lui attribue une qualité humaine, celle de l'erreur. Dieu n'est plus une force omnisciente, omnipotente, ni même la figure de la perfection. Dieu est un peintre qui s'est trompé, Dieu est un peintre comme Cornélius, Dieu devient ni plus ni moins un confrère dont on commente le travail.

Pour Cornélius, il semble y avoir eu un transfert de la notion de sacré. Le sacré n'est plus en Dieu, mais dans la nature, dans des paysages inaccessibles à l'homme: «il rêvait à des campagnes tremblantes de rosées, plus belles que les bords de l'Anio crépusculaire, mais désertes, trop sacrées pour l'homme» (p. 1216). C'est dans le rêve que Cornélius se réfugie, c'est là qu'il retrouve le sens du sacré qui ici se teinte d'un caractère libidinal: la campagne féminine est tremblante et belle. Pour Cornélius, le sacré reste accessible par l'exil, par le retrait du monde et c'est l'humain qui est aussi visé par la désacralisation. La sottise des hommes est, en effet, mise en opposition avec les paysages; elle contraste avec les forces de la nature "des expressions d'avarice, de sottise ou de férocité notées sous tant de beaux ciels» (p. 1217). Cornélius devant son incapacité à peindre garde un attachement sensible aux objets et non à l'humain:«Ses mains déformées avaient, en touchant les objets qu'il ne peignait plus, toutes les sollicitudes de la tendresse» (p. 1216).

Il est ici important de tenir compte du moment historique dans lequel évolue Cornélius. Cornélius est un ancien disciple de Rembrandt. Le texte évoque même une similitude entre des scènes peintes par Cornélius et un tableau de Rembrandt: La leçon d'anatomie du docteur Tulp. Ce tableau fut peint par Rembrandt en 1632.

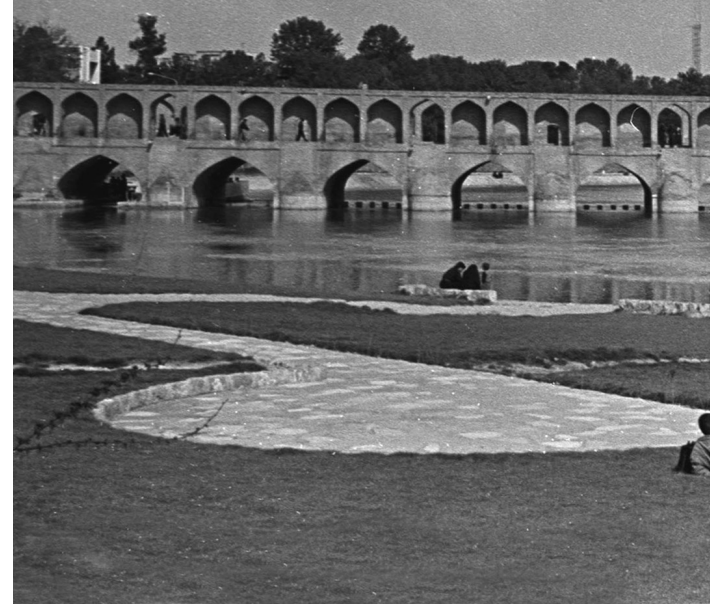

En lisant ces lignes: «Le beau corps gras de son modèle Frédéric Gerritsdochter, étendu sur la table d'anatomie» (p. 1217), on ne peut s'empêcher de faire le rapprochement entre le tableau de Rembrandt et la perception du monde selon Cornélius. Cette référence picturale est un intertexte significatif même s'il ne fait qu'effleurer le texte. Ce tableau du maître a visiblement impressionné l'élève Cornélius et ce n'est pas un hasard si ce tableau marque un moment charnière dans l'histoire de l'art sur la question du rapport de l'art avec le religieux et le sacré. Comme l'explique Jean-Marie Pontevia, dans ce tableau de Rembrandt, le cadavre est traité comme objet, ce qui représente le début, dans l'histoire de l'art occidental, d'une désacralisation de la mort. La mort est représentée d'un point de vue profane:

Désacralisation progressive de l'image de la mort. En fait, il faut parler d'une perte de transcendance [...] Ce qui se 


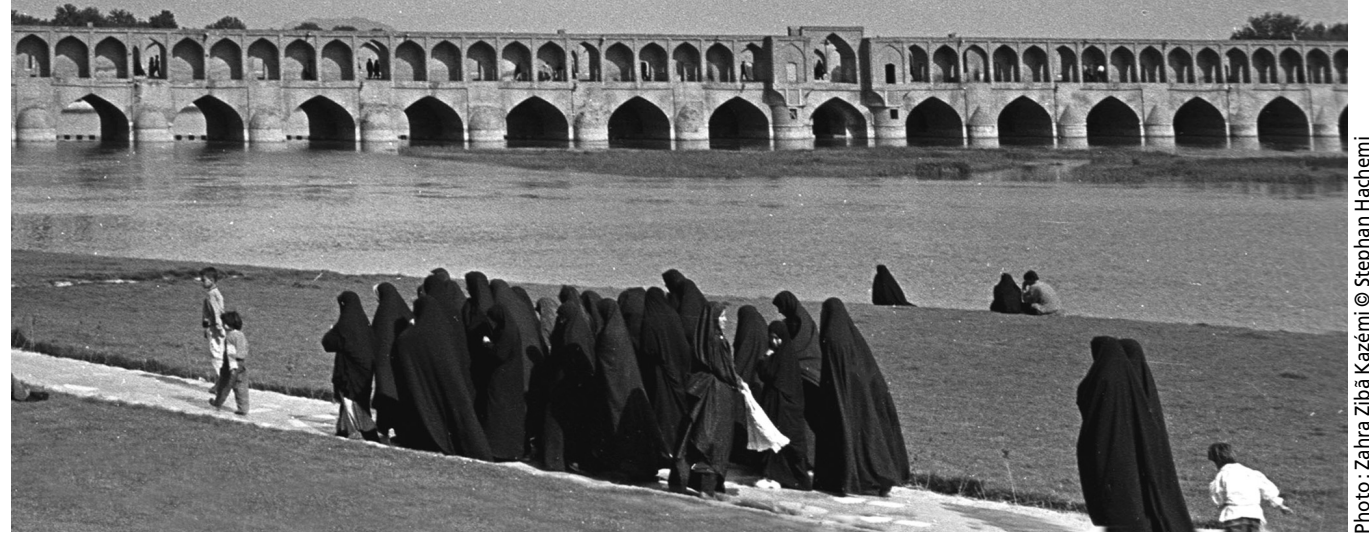

Dieu constituait le principe de l'identité alors que le monde terrestre était le lieu du changement et de l'altérité où l'identité est toujours compromise. Lorsque la référence théologique qui sous-tend le principe d'identité se trouve remise en question, il y a perte d'identité: «[...] dès que la référence théologique cesse de garantir la vérité et la plénitude du sens, s'ouvre la question de l'identité, l'identité comme question [...] dès que le garant absolu de l'identité du moi disparaît à l'horizon de la conscience, la notion d'identité se volatilise » (Pontévia, 1986, p. 42).

En remettant Dieu en question, Cornélius pose le problème de sa propre identité. Sa perception du monde bascule et il se trouve dès lors dans l'impossibilité de créer. Créer appelle un positionnement du sujet, ce que Cornélius n'est plus en mesure d'instaurer. Cornélius ayant évacué Dieu, le garant du sens transcendant, est à la recherche d'une autre instance absolue qu'il n'arrive plus à saisir et donc qu'il ne peut traduire dans sa peinture. Cette instance est figurée par l'élément humide: «une lumière jaunâtre emplissait la chambre; la pluie lavait humblement les vitres; l'humidité était partout. L'élément humide enflait sous forme de sève la sphère grumeleuse de l'orange, boursouflait la boiserie qui criait un peu, ternissait le cuivre du pot» (p. 1216). Lélément humide est envahissant, il pénètre, englobe et transforme; il donne sens, mais il est porteur d'un sens que Cornélius ne peut pas rendre dans ses tableaux.

L'humide donne son sens aux objets perçus par le peintre, mais il est aussi un rappel du médium, il est donc doublement enveloppant. Les fluides sont les éléments de l'expérience picturale. Le peintre est instigateur du processus d'imprégnation de deux corps qui se compénètrent: la toile comme surface d'absorption et la peinture comme liquide qui se répand sur cette surface. Le peintre est confronté à la matérialité de son médium. Il expérimente par l'interaction toile-peinture, des phénomènes de perméabilité, de capillarité, d'osmose, de symbiose. De l'élément fluide qu'est la peinture à l'eau comme élément et principe de vie, il n'y a qu'un pas:

Traiter la couleur [peinture] comme un fluide, c'est la traiter comme l'eau [...] aussi voit-on ressurgir ici l'idée extravagante par laquelle, remarque Nietzsche, s'inaugure la pensée grecque: la thèse selon laquelle l'eau serait l'origine et la matrice de toutes choses. Tout est eau première manière de dire Tout est Un, désignation de l'élément humide comme substance de tout ce qui est, et spécialement de tout ce qui vit (Pontévia, 1986, p. 251). 
Cornélius est impuissant à reproduire l'humide donc à reproduire la vie: «[il] renonçait à reproduire sur la toile cette double coulée humide et lumineuse imprégnant les choses et embuant le ciel » (p. 1216). Incapable d'établir l'humide et donc la vie comme porteur du sens transcendant, Cornélius ne peut que s'enfoncer plus profondément dans sa mélancolie. Cornélius ne peut plus rendre intelligible la vie qu'il perçoit dans le monde tout comme il ne peut plus se comprendre lui-même car son cœur est habité par l'humide: «Ce vieillard, que la misère semblait gonfler, paraissait atteint d'hydropisie du cœur» (p. 1216). Comme l'humide boursouflait les boiseries, la misère gonfle le cœur de Cornélius. Cornélius perçoit le spectacle de la misère et du cru de l'humanité qui détonne sur la beauté de la nature et c'est ce qui lui fait dire que Dieu s'est trompé.

\section{COMMENT WANG-FÔ FUT SAUVÉ}

Comment Wang-Fô fut sauvé nous présente le vieux peintre chinois Wang-Fô et son disciple Ling. Depuis leur rencontre dans une taverne, les deux hommes se sont liés d'amitié. Petit à petit, Ling fait son apprentissage auprès de Wang-Fô. Il apprend à mélanger la couleur et avec l'apprentissage de la technique du maître vient aussi l'apprentissage d'une vision du monde. Ling délaisse peu à peu sa vie d'avant où il vivait dans le confort de la richesse avec sa femme et il expérimente un changement intérieur profond où ses valeurs et sa vision du monde se transforment sous l'influence $\mathrm{du}$ peintre. Ling apprend à percevoir le réel autrement: "Wang-Fô venait de lui faire cadeau d'une âme et d'une perception neuves» (p. 1145). Après le décès de sa femme, Ling vend ses biens et laisse sa maison pour partir sur les routes avec Wang-Fô. L'empereur Han, menacé par la fascination que le peintre exerce sur son peuple, fait arrêter Wang-Fô. Ling est tué par des soldats en tentant de sauver son maître. Wang-Fô est condamné mais la sentence ne sera exécutée que lorsque le peintre aura complété un tableau pour l'empereur. C'est en peignant ce tableau que Wang-Fô se fond à son œuvre. Ling apparaît alors sur la mer que Wang-Fô peint et il vient chercher son maître pour fuir sur une barque alors que le palais de l'empereur est inondé par la mer.

Comme nous l'indique Marguerite Yourcenar dans le post-scriptum des Nouvelles orientales, Comment Wang-Fô fut sauvé s'inspire d'un apologue taoïste de la vieille Chine. En Chine, l'art pictural est intimement lié à la philosophie taoïste, la peinture est un art de vivre tout comme le $\mathrm{TaO}^{2}$.

Dans cette nouvelle, nous nous sommes particulièrement intéressés au regard ou à la métaphore de l'œil, l'œil étant par extension une référence à l'art visuel et le personnage principal étant un peintre. Elena Pessini, dans son article L'artiste dans l'œuvre de Marguerite Yourcenar, souligne la relation étroite entre le regard et le sacré: «Sa méthode [celle de Wang Fô] fascine les hommes qu'il rencontre et son disciple Ling voudra franchir les portes de son immense savoir, être initié à un rite où l'accès au sacré se fait par le regard» (Pessini, 1990, p. 6). Pessini vise juste, mais nous croyons qu'il est nécessaire de porter plus loin la réflexion. Le regard est l'accès au sacré car c'est par le regard que Wang-Fô porte sur le réel que Ling fait son apprentissage et c'est aussi par le regard que se vit l'intensité des émotions pour Ling: "Ling désespéré regardait son maître en souriant, ce qui était pour lui une façon plus tendre de pleurer» (p. 1147). Le regard de Wang-Fô peut se lire comme le regard de l'accompagné. Wang-Fô, fort de l'expérience d'une vie et de son travail de peintre sur la perception du réel, transmet à Ling, l'accompagnant, une vision de la vie.

\section{L'ACCOMPAGNANT FAIT L'EXPÉRIENCE D'UNE RÉVÉLATION}

\section{À UNE NOUVELLE PERCEPTION DU RÉEL.}

\section{ACCOMPAGNANT ET ACCOMPAGNÉ PEUVENT TOUS DEUX}

\section{RESSENTIR UN AILLEURS SACRÉ, UN AILLEURS}

CHARGÉ D'UN SENS INNOMMABLE

\section{QUI DÉPASSE LA PERCEPTION USUELLE DE LA VIE.}

Dès le début du texte, on remarque que Wang-Fô est le détenteur du regard, du regard au sens du Tao, c'est-à-dire un regard qui a le pouvoir de voir dans l'invisible. Le regard de Wang-Fô est celui qui voit le monde tel qu'il est réellement, il voit au-delà de la réalité. Son regard sur le monde, Wang-Fô le traduit dans son œuvre. Le rapport qu'il a avec sa création est aussi une métaphore sur sa manière de décoder et d'appréhender le monde réel. Comme c'est le cas des personnages créateurs chez Yourcenar, la création est une traduction d'une vision du monde. Wang-Fô par son œuvre donne accès à un autre royaume, à son royaume d'images qui fait l'envie de l'empereur:«[...] puisque tes yeux, Wang-Fô, sont les deux portes magiques qui t'ouvrent ton royaume. Et puisque tes mains sont les deux routes aux dix embranchements qui te mènent au cœur de ton empire»
Hennezel en se référant au témoignage de Jean-Dominique Bauby; c'est la perception et l'intensité de l'instant qui changent:

Il est alors extraordinairement dans l'instant présent, un être de perceptions, de sensations, bien qu'il soit totalement paralysé. Toutes les petites choses du quotidien sont habitées d'une intensité et d'une présence inouïes. En fait je crois que lorsqu'on s'approche de ses derniers instants on devient contemplatif (De Hennezel et Leloup, 2000, p. 110).

Pour bien peindre, Wang-Fô doit s'harmoniser à son sujet: "Une nuit, dans une taverne, il [Ling] eut Wang-Fô pour compagnon de table. Le vieil homme avait bu pour se mettre en état de mieux peindre un ivrogne» (p. 1144). Contempler, c'est aussi vivre à la place de l'autre, ressentir ce que l'autre ressent, s'imprégner d'émotions pour 
donner vie à l'image. Wang-Fô, qui est perçu tantôt comme un sage et tantôt comme un sorcier, est porteur du pouvoir de donner la vie à ses représentations du monde: «On disait que Wang-Fô avait le pouvoir de donner la vie à ses peintures (portraits) par une dernière touche de couleur qu'il ajoutait à leurs yeux» (p. 1145). Cette vie dépasse l'image du tableau et prend un sens particulier pour ceux et celles qui regardent l'œuvre, ce qui donne à Wang-Fô un pouvoir sur les uns et les autres. Il est craint et vénéré, il provoque la fascination comme celui qui détient un secret, le secret de la vie et le secret d'un royaume qui s'étend au-delà de la vie. Son pouvoir est à la fois celui du devin et celui du maître qui appelle la mort: «Ensuite, Wang-Fô la [la femme de Ling] peignit en costume de fée parmi les nuages du couchant, et la jeune femme pleura, car c'était un présage de mort» (p. 1145).

\section{LING, DISCIPLE ET ACCOMPAGNANT}

Ling est le disciple de Wang-Fô et le premier à bénéficier de sa présence. Ling est initié à la perception du monde selon Wang-Fô et il le dit lui-même; Wang-Fô lui a donné une âme neuve. Cette âme neuve, Ling la découvre et la façonne au côté de son maître. Au fil des jours, l'âme s'affine et avec elle la sagesse et le détachement du monde matériel. Comme l'accompagnant, Ling peut bénéficier d'une présence qui l'invite à réfléchir sur le monde et l'interprétation qu'il en fait. Ling, à côtoyer son maître, devient lui-même sensible à une autre réalité par la contemplation. Ling apprend par l'exemple et, comme son maître, il devient contemplatif. La contemplation est une étape cruciale du mourir; elle permet à l'accompagné de retrouver la sérénité et une certaine paix face à la mort; elle permet un détachement et donne accès à l'univers au-delà de la vie en développant une nouvelle perception du réel.

Pour Ling, accompagner son maître est une préoccupation constante et se vit au quotidien:

Ling [...] veillait sur le sommeil du maître et profitait de ses extases pour lui masser les pieds. Au point du jour, quand le vieux dormait encore, il partait à la chasse de paysages timides dissimulés derrières des bouquets de roseaux. Le soir, quand le maître, découragé, jetait ses pinceaux sur le sol, il les ramassait. Lorsque Wang était triste et parlait de son grand âge, Ling lui montrait en souriant le tronc solide du vieux chêne; lorsque Wang était gai et débitait des plaisanteries, Ling faisait humblement semblant de l'écouter (p. 1146).
Ling accompagne spirituellement son maître, dans une sphère physique. Cette proximité physique souligne l'abandon de l'un à l'autre et elle n'est pas sans rappeler l'entente tacite qui unit accompagnant et accompagné dans une complicité physique si signifiante tant pour l'un que

\section{LA MORT EST PEUT-ÊTRE}

\section{AINSI UN TABLEAU, \\ L'APOGÉE DE L'CEUVRE \\ QU'EST LA VIE.}

pour l'autre: «Le vieux s'enveloppa dans des loques et Ling se coucha contre lui pour le réchauffer» (p. 1146).

La femme de Ling, qui était pour lui un miroir et un talisman, est remplacée par une peinture d'elle réalisée par Wang-Fô. Ce miroir qu'était sa femme, Ling n'en a maintenant plus besoin car les paysages de Wang-Fô deviennent son miroir. La nature telle que Wang-Fô la représente dans son art et telle qu'il la fait découvrir à Ling n'est pas simplement extérieure mais aussi un reflet de lui-même. Comme accompagnant, Ling se découvre lui-même par un effet de miroir du peintre Wang-Fô et la contemplation mène Ling à un retour sur soi et à une nouvelle interprétation du monde, qui devient alors esthétique et pictural. Pour les peintres chinois, la nature est plus qu'une représentation du réel: "Cette dernière [la nature] n'est pas simplement envisagée comme un cadre extérieur ou un terme de comparaison; elle tend à l'homme une miroir fraternel lui permettant de se découvrir et de se dépasser, les figures extérieures deviennent la représentation d'un monde intérieur»(Cheng, 1991, p. 140). Découvrant cette nouvelle perception, Ling se révèle à lui-même et dépasse sa condition de simple mortel en ayant accès par son maître à une autre vision du réel où se met en place une harmonie parfaite entre le réel et la peinture. Cet enchevêtrement du réel et du pictural est souligné tant par l'action des personnages que par les descriptions détachées que ceux-ci font du réel. Ainsi, grâce à l'influence du peintre, Ling vainc ses peurs, celles du tonnerre, des insectes et du visage des morts. Tous ces éléments font dorénavant partie d'une représentation visuelle et artistique. À la mort de sa femme, Ling, préoccupé par son travail d'assistant du maître, oublie de verser des larmes car il est sentimentalement détaché de sa femme et il la perçoit simplement comme le sujet d'un tableau. La distance créée par une vision picturale du réel a provoqué le détachement: "Un matin, on la trouva pendue aux branches du prunier rose. [...] Wang-Fô la peignit une dernière fois car il aimait cette teinte verte dont se recouvre la figure des morts. Son disciple Ling broyait les couleurs, et cette besogne exigeait tant d'application qu'il oubliait de verser des larmes»(p. 1145). Ling parce qu'il réussit à vaincre sa peur des morts vainc aussi la peur de sa propre mort, ce qui lui permet de devenir immortel et de faire le passage sans contrainte du monde du réel à celui de l'invisible; il deviendra même le passeur de Wang-Fô. Ling est l'accompagnant idéal: il accompagne Wang-Fô aussi bien dans la vie, comme disciple, que dans la mort. Ling meurt avant son maître et c'est lui qui accueille le peintre dans l'autre monde.

Le passage du monde réel à la mort ou au monde de Wang-Fô est clairement présenté dans une remarque que Ling fait au sujet de l'empereur Han et ses courtisans: "Ces gens ne sont pas faits pour se perdre à l'intérieur d'une peinture» (p. 1153). Ces quelques mots résument toute la conception $\mathrm{du}$ monde selon Wang-Fô et la relation à l'art pictural qui sous tend l'ensemble du texte. Le passage au monde de l'invisible se fait par l'art et c'est la conception de l'art pictural chinois qui s'exprime ici. Une peinture réussie, selon les critères de cet art, est celle qui suscite chez l'observateur et chez le peintre le désir d'y vivre. Se fondre dans l'œuvre est le véritable dépassement, l'accomplissement ultime, le but visé: «La peinture doit susciter, en celui qui la contemple, le désir de s'y trouver [...] L'homme finira par s'absorber dans l'œuvre, car là est pour lui le véritable dépassement, là est la participation au parachèvement de la Création»(Cheng, 1991, p. 102-148). Le peintre chinois par son œuvre participe à la création et c'est le geste divin qu'il imite et recrée ainsi: "le trait tracé, aux yeux du peintre chinois, est réellement le trait d'union entre l'homme et le surnaturel. Car le trait, par son unité interne et sa capacité de variation, est Un et Multiple. Il incarne le processus par lequel l'homme dessinant rejoint les gestes de la Création »(Cheng, 1991, p. 73).

Le processus de création tel qu'il est conçu par l'art chinois voit l'achèvement d'un tableau comme l'apogée de l'œuvre. La mort est peut-être ainsi un tableau, l'apogée de l'œuvre qu'est la vie:

Concevoir le papier vierge comme le Vide originel par où tout commence, le premier trait tracé comme l'acte de séparer le Ciel et la Terre, les traits qui suivent et qui engendrent au fur et à mesure toutes formes comme de multiples métaphores de premier trait, et enfin, l'achèvement du tableau 
comme le degré suprême d'un développement par lequel les choses retournent au Vide originel (Cheng, 1991, p. 106).

Le concept du Vide est une des bases de la pratique de l'art pictural chinois. Le peintre doit dans son œuvre arriver à un parfait équilibre entre le Vide et le Plein, entre le Visible et l'Invisible: "C'est bien le Vide qui permet le processus d'intériorisation et de transformation par lequel toute chose réalise son même et son autre, et par là atteint la totalité. En ce sens, la peinture en Chine est pleinement une philosophie en action; elle y est envisagée comme une pratique sacrée»(Cheng, 1991, p. 48). Le Vide, en même temps qu'il est l'accès à l'invisible ou sa représentation, bouleverse la perception linéaire. Le Vide établit ainsi une relation de devenir réciproque entre le peintre et la nature et entre le spectateur et le tableau. On a affaire dans l'art chinois à une double perspective qui transforme la relation sujet-objet. Le peintre en général jouit d'une vision globale en même temps qu'il semble se mouvoir dans le tableau, il contemple ainsi un espace de loin, de près et de différents côtés: "Le mouvement d'éloignement dans l'espace est en fait un mouvement circulaire qui revient et qui par le renversement de la perspective et du regard, transforme la relation $d u$ sujet-objet (le sujet se projetant, par degrés, au-dehors; et le dehors devenant le paysage intérieur du sujet)»(Cheng, 1991, p. 105). Cette conception de l'espace qui découle de la double perspective est aussi intimement liée à la conception du temps selon le taoïsme. Cette notion d'un espace multiple et différent introduit un nouveau rapport espace-temps fondé sur l'affirmation du Vide du Tao:

Si le temps est perçu comme actualisation de l'Espace vital, le Vide, en introduisant la discontinuité dans le déroulement temporel, ré-investit, en quelque sorte, la qualité de l'Espace dans le Temps, assurant ainsi le rythme juste des souffles et l'aspect total des relations. Ce changement qualitatif du temps en Espace est la condition même d'une vraie vie qui ne soit pas unidimensionnelle

(Cheng, 1991, p. 67).

La mort de Wang-Fô est tout à fait la représentation de ce changement du temps en Espace. Wang-Fô se sauve de sa condamnation à mort en mettant le pied dans l'univers pictural qu'il a créé, la réalité bascule ici dans l'imaginaire visuel: la peinture sort du cadre, l'eau que Wang-Fô a peint noie l'empereur et inonde son royaume. Traversant de la réalité du monde à la réalité du tableau, Wang-Fô et Ling ont accès à un autre espace, un espace hors du temps et infini, le temps non chronologique: «le peintre Wang-Fô et son disciple Ling disparurent à jamais sur cette mer de jade bleu que Wang-Fô venait d'inventer» (p. 1153). Accompagnant et accompagné font ici le saut hors du temps.

\section{QUÊTE DE LA TRANSCENDANCE ET QUÊTE DU BEAU: LES PASSAGES OBLIGÉS DE LA MORT}

Ces deux nouvelles de Marguerite Yourcenar appartiennent à des genres différents. Comment Wang-Fô fut sauvé est une légende et la Tristesse de Cornélius Berg est un récit bref. Le rapport imaginaire-réel est exploité différemment dans la légende et dans le récit. La légende laisse libre cours à un imaginaire où le souci du réalisme est délaissé au profit du merveilleux.

Comme nous l'avons vu plus haut, la tristesse de Cornélius est un sentiment révélateur du dépressif et de la blessure narcissique. Pour Kristeva, l'objet idéal ou la beauté pourrait avoir la capacité de supprimer l'humiliation de la blessure narcissique qui sous-tend le désespoir du dépressif. Le beau serait cet objet idéal qui ne déçoit jamais la libido et serait le réparateur absolu et indestructible de l'objet abandonnique. Le beau serait cet idéal, cet au-delà qui dépasse la mort. Nous avons ici affaire à la dynamique de la sublimation. Par la sublimation, le dépressif crée un hyper-signe. L'homme transcende ainsi la douleur d'être séparé. Par l'allégorie, le dépressif donne de la magnificence à ce qui n'est plus. Par une signification supérieure, le dépressif peut refaire le néant dans une harmonie inaltérable. Le bel objet est la marque d'une adhésion au sublime:

La sublimation seule résiste à la mort.

Le bel objet capable de nous envoûter

dans le monde nous paraît plus digne

d'adhésion que toute cause aimée ou

haïe de blessure ou de chagrin... mais

cette adhésion au sublime n'est plus

libidinale... La beauté est artifice,

elle est imaginaire (Kristeva, 1987,

p. 111-112).

Cette beauté imaginaire et cette capacité imaginaire rendent possible un transfert du sens au lieu même où il s'est perdu: "L'imaginaire est miracle, mais il est en même temps sa pulvérisation: auto-illusion, rien que du rêve et des mots, des mots, des mots» (Kristeva, 1987, p. 115).

Le beau est de près lié à l'art visuel. Jean-Marie Pontévia dans Tout a peut-être commencé par la beauté remonte à l'art grec, aux origines de la notion du beau, et nous explique les liens qui unissaient beauté et divin. Le beau chez les Grecs anciens est un mot de la même famille que phénomène (apparence) et épiphanie (révélation, manifestation). Le beau est éclat de l'apparence.
La beauté est cette apparence qui retient l'attention, elle est le signe d'un ordre, d'une harmonie. La beauté est le signe du bien. Le bien est le principe de l'ordre ontologique; c'est Dieu. Le bien ou le beau mesure la perfection de l'apparence. Le bien est au-delà de l'être, il est de l'ordre de la perfection et de l'immuable. Le divin est ce qui en changeant reste ce qu'il est. La beauté apaise et transporte, la beauté est accord et la laideur est discordance à l'égard du divin. L'art parce qu'il déjoue le vieillissement est la représentation du divin. Le divin est la permanence du beau, une constance de l'apparence. La statuaire et les temples grecs sont autant de signes du divin car ils représentent le beau immuable. L'harmonie est l'accord qui crée la Beauté, laquelle est dans le monde la manifestation du divin.

Dans Comment Wang-Fô fut sauvé, c'est par l'apprentissage de Ling que le lecteur découvre l'art du maître. Ling broie les couleurs et apprend à regarder le monde selon Wang-Fô. Ling découvre la beauté et la cruauté du monde disparaît car Wang-Fô transforme le réel par l'art. Découvrant la beauté et la contemplation de la beauté du monde, Ling apprivoise la mort; la sienne et celle de son maître.

Le peintre, tant Wang-Fô que Cornélius, travaille à affiner sa peinture et affiner aussi sa capacité à voir au-delà de la réalité matérielle. Il perçoit une autre dimension, celle du beau, celle de la couleur et de l'art. L'harmonisation des couleurs et des formes est le travail du peintre. La réalité se charge d'un autre sens grâce à ce travail d'affinement de la perception du monde. Les deux personnages de Yourcenar travaillent à perfectionner l'acuité de leur regard et c'est en qualité de peintre qu'ils perçoivent une autre dimension ou donnent une autre interprétation du monde réel.

Ce travail sur la perception du monde est, pour Wang-Fô, soutenu par une philosophie et c'est cette philosophie qui fait défaut à Cornélius. Le tao est indissociable de l'art pictural chinois. Pour l'artiste chinois, le travail sur l'œuvre est aussi un travail sur soi. C'est dans l'œuvre même et donc en soi que l'artiste trouve un sens, celui d'une harmonisation transcendante:

Celle-ci [l'œuvre] repose, en vérité, sur le principe de la discipline et de la vie: par l'Un, maîtriser la Multiplicité; à partir de la Multiplicité maîtriser l'Un. Elle ne recourt ni à la montagne, ni à l'eau, ni au pinceau, ni à l'encre, ni aux anciens, ni aux modernes, ni aux saints. Elle est l'œuvre véritable, celle qui se fonde sur sa propre substance (Cheng, 1991, p. 105).

La substance est ce qui manque à Cornélius, celui-ci est incapable de saisir la 


\section{LE CATALYSEUR, VOIRE LE DÉCLENCHEUR}

\section{DE LA CONTEMPLATION CHEZ L'ACCOMPAGNÉ.}

substance présente dans l'humide. L'humide pourrait donner un sens transcendant à la matière si le peintre pouvait la rendre sur la toile. Mais Cornélius trouve la transcendance ou un sentiment de sacré uniquement dans le rêve; l'imaginaire pictural de Cornélius ne franchit pas les frontières du réel. Cornélius est un personnage qui reste dans le monde et subit les marques du temps linéaire, Cornélius vieillit et la matière a raison de lui. Il est condamné à la dépression, à la mort et la sublimation dans l'imaginaire ne le sauve pas mais creuse plutôt l'écart entre le réel et le beau. Cornélius est incapable de se réfugier dans le beau et c'est l'abject du monde qui lui saute aux yeux. Vivant dans une époque où le sacré s'exprime par le religieux plutôt que dans la substance même de l'art pictural, Cornélius est confronté au néant, au non-sens. Ce néant est l'invisible, mais ce néant est aussi pour Cornélius, le néant de l'être, d'où l'être dépressif. Car l'art visuel est le médium du questionnement de l'invisible et de l'être.

En Chine, peindre est un art de vivre. L'art pictural révèle le mystère de l'univers. La peinture en Chine est considérée comme une pratique sacrée:

En Chine, de tous les arts, la peinture occupe la place suprême. Elle est l'objet d'une véritable mystique [...] la peinture représente une manière spécifique de vivre. Elle vise à créer, plus qu'un cadre de représentation, un lieu médiumnique où la vraie vie est possible. En Chine, l'art et l'art de la vie ne font qu'un (Cheng, 1991,

p. 11-12).

Le principe du Vide est une pièce mâ̂tresse de la philosophie taoïste. Ce Vide est à mettre en opposition avec le Rien de la tradition occidentale. Dans la tradition occidentale, le Rien est Néant, tandis que dans la philosophie orientale le concept de Néant est inexistant car le Vide est aussi le Plein. Le Vide n'existe jamais seul si bien que le Vide est le point de rencontre du manque et de la plénitude, du même et de l'autre.

Le rapport sujet-objet est court-circuité chez Cornélius et c'est ce qui le rend incapable de peindre. Le rapport au monde de Cornélius est aussi un rapport de retrait ou de marge, Cornélius a l'œil critique face aux humains qui l'entourent et il tend vers une certaine misanthropie et une grande solitude. Contrairement à Wang-
Fô, il n'est pas accompagné d'un disciple, mais il est plutôt le disciple abandonné à lui-même. On peut croire que Cornélius ne se perçoit plus que comme sujet ce qui l'entraîne vers le Néant. La difficulté de Cornélius semble résider dans l'identité et la représentation de soi à travers son art, c'est-à-dire une difficulté à saisir ou comprendre ou même sentir le je me vois me voir (corrélation essentielle de la conscience dans sa relation à la représentation) propre à l'art visuel et qui devrait aboutir à une réappropriation de soi et de l'être pour le peintre.

Ce je me vois me voir est peut-être aussi un des fondements de l'accompagnement où le rapport sujet-objet pourrait se lire comme le rapport de l'accompagnant à l'accompagné. L'accompagnant est le miroir, le catalyseur, voire le déclencheur de la contemplation chez l'accompagné. Le rapport à l'autre dans la mort mène à une projection de soi dans l'ailleurs tant pour l'accompagné que pour l'accompagnant. La représentation ou le spectacle de la mort, comme un tableau, est cathartique pour l'accompagnant qui vit ainsi, par procuration, une expérience transcendante. L'accompagnant fait l'expérience d'une révélation à une nouvelle perception du réel. Accompagnant et accompagné peuvent tous deux ressentir un ailleurs sacré, un ailleurs chargé d'un sens innommable qui dépasse la perception usuelle de la vie. Le rapport à l'autre dans la mort s'oppose à la solitude de la mort, car seul, non accompagné, l'être est confronté à lui-même face à une conscience qui tourne à vide, un sujet qui n'a aucune prise sur un objet. Le rapport à l'autre, le rapport accompagnant-accompagné se vit chez Wang-Fô dans une intimité et une complicité exprimée au-delà des mots. L'accompagnant dans le rapport à l'autre, dans une compréhension tacite, sert d'appui vers la transcendance pour l'accompagné, il est le marchepied qui permet le saut. Wang-Fô, accompagné par Ling, réussit à transmettre à son disciple son état contemplatif et Ling, qui meurt accidentellement avant son maître, tend la main à Wang-Fô pour que continue leur voyage dans le monde infini symbolisé par une barque peinte sur la mer.

Ces deux peintres, Wang-Fô et Cornélius, nous font la démonstration d'une fin de vie. Wang-Fô réussit son passage accompagné d'un disciple à qui il a pu transmettre une vision autre du réel et une philosophie de vie exempte de peur et axée sur une harmonie picturale empreinte de détachement. Cornélius, peintre de l'identité, se dirige vers sa mort en solitaire et en quête de sens. Mourir est peut-être un art, celui de l'apprentissage d'une nouvelle perception, et la mort n'est peut-être qu'une frontière imaginaire. Tout comme l'art, mourir pourrait être la capacité de donner un autre sens au réel, le pouvoir de le transcender, et accompagner serait la leçon de transcendance, une anatomie du sacré.

\section{Bibliographie}

CHENG, François (1991). Vide et plein, le langage pictural chinois, collection «Points essais », Paris, Seuil.

DE HENNEZEL, Marie et Jean-Yves LELOUP (2000). L'art de mourir, traditions religieuses et spiritualité humaniste face à la mort, collection «Pocket», Paris, Robert Laffont.

KRISTEVA, Julia (1987). Soleil noir, dépression et mélancolie, Paris, Gallimard, 265 p.

PESSINI, Elena (1990). L'artiste dans l'œuvre de Marguerite Yourcenar, Actes du colloque: Marguerite Yourcenar et l'art, l'art de Marguerite Yourcenar, Tours, Université de Tours, Société internationale d'études yourcenariennes.

PONTÉVIA, Jean-Marie (1986). Ogni dipintore dipinge sè, écrits sur l'art et pensées détachées tome III, Bordeaux, William Blake \& Co.

YOURCENAR, Marguerite (1982). EEuvres romanesques, coll. "Bibliothèque de $\mathrm{La}$ Pléiade», Paris, Gallimard, 1257 p.

\section{Notes}

1. Le rituel de dormition tel qu'il est présenté par Jean-Yves Leloup dans le livre L'art de mourir est un essai de synthèse de différents rituels connus et pratiqués dans les monastères russes, grecs, égyptiens, roumains, français orthodoxes et il compte sept étapes: la Compassion, l'Invocation, l'Onction, l'Écoute, le Pardon, la Communion, la Contemplation (De Hennezel et Leloup, 2000, p. 94).

2. Le Tao: le sens du mot chinois est chemin ou voie; il peut aussi être considéré comme le principe régulateur de l'alternance du Ying et du Yang; le Ying et le Yang se substituent tour à tour l'un et l'autre ou subsistent simultanément dans une relation d'opposition.

3. Afin de faciliter la lecture et l'analyse du texte, nous indiquons ici la référence pour l'ensemble des citations tirées des deux nouvelles des Nouvelles Orientales (Yourcenar, 1982). 